\title{
Measurement of adult vocal fold length
}

\author{
Mao-Chang Su, M.D., Te-Huei Yeh, M.D.", Ching-Ting Tan, M.D., Ph.D, , Chia-Der Lin, M.D.", \\ Oan-Che Linne, M.D.", Shiann-Yann Lee, M.D.*
}

\begin{abstract}
Laryngeal photographs from 165 Taiwanese subjects were taken during microlaryngoscopy. Photographs of the vocal fold during the open phase were chosen. For measuring the vocal fold length, both the photograph of a ruler and the larynx were taken under the operating microscope, thus forming a 'new scale'. Photographs of the vocal fold and the ruler were then processed at the same power of magnification. The length of the vocal folds was measured with the 'new scale'. In 100 patients receiving direct laryngoscopy under neuroleptic anaesthesia, the vocal fold length was $14.6 \pm 1.2 \mathrm{~mm}(\mathrm{n}=35)$ for males and $11.1 \pm 1.0 \mathrm{~mm}(\mathrm{n}=65)$ for females. In 65 patients under general anaesthesia, the vocal fold length was $15.3 \pm 1.6 \mathrm{~mm}(\mathrm{n}=23)$ for males and $13.5 \pm 1.3 \mathrm{~mm}(\mathrm{n}=42)$ for females. Statistical analysis revealed a significant difference between both groups and both sexes. Elongation of the vocal fold under general rather than neuroleptic anaesthesia may be important when performing functional surgery of the vocal fold.
\end{abstract}

Key words: Laryngoscope; Vocal Fold; Adult

\section{Introduction}

To obtain an accurate measurement of the human vocal fold length has been a difficult task. So far, the available data were either directly measured from cadavers or estimated from X-ray films of living humans. ${ }^{1,2}$ In both cases, no precise vocal fold length was obtained. We have developed an approach that allows measurement of the length of the adult vocal fold.

\section{Materials and methods}

For the past three years, photographs of the vocal fold were taken during microlaryngoscopy from patients with unilateral benign vocal fold lesions. To avoid the bias originating from pathological changes, only the healthy side was measured. In 165 patients, there were 58 males (age range 24-83 years) and 107 females (age range 20-67 years). Peroral laryngoscopy was performed under neuroleptic anaesthesia in 100 patients (35 males, 65 females). Surface anaesthesia was attained by spraying 5 per cent cocaine solution topically. Then, intravenous injections of atropine $(0.2 \mathrm{mg})$, diazepam $(10 \mathrm{mg})$, and meperidine $(50 \mathrm{mg})$ were given. Blood pressure and EKG were monitored. In this condition, the patient was relaxed, free from pain and able to maintain his reflex abilities. If requested, the patients were able to phonate voluntarily or take a deep breath during the procedure. The motion of the vocal fold could be demonstrated by direct laryngoscopy.

In another 65 patients (23 males, 42 females), laryngoscopy was performed under general anaesthesia by peroral endotracheal intubation (tube size $6 \mathrm{~mm})$. The patients were totally paralysed. No motion of the vocal fold could be observed. During the procedure, a camera connected to the side aperture of the surgical microscope was used to photograph the vocal fold. All images were magnified $25 \times$ and the focus depth of the lens was $400 \mathrm{~mm}$. A ruler was also photographed at the same magnification and distance to form a 'new scale'. Photographs of the vocal folds and the ruler were then developed and enlarged simultaneously. The length of vocal folds was measured with the "new scale' by two researchers independently. To perform a consistent measurement, some reference lines were taken as shown in Figure 1. This figure of the vocal fold was transformed from a true photograph which represents a very common laryngeal picture in our patients. The size of the arytenoid process is usually negligible and this is generally the case in the Taiwanese population. Line $\mathrm{A}$ is the inner border of the vocal process. Line B is the line connecting the bilateral vocal processes. Line $\mathrm{C}$ is the median line bisecting line $\mathrm{B}$ perpendicularly. There was no difficulty in identifying the arytenoid process under 


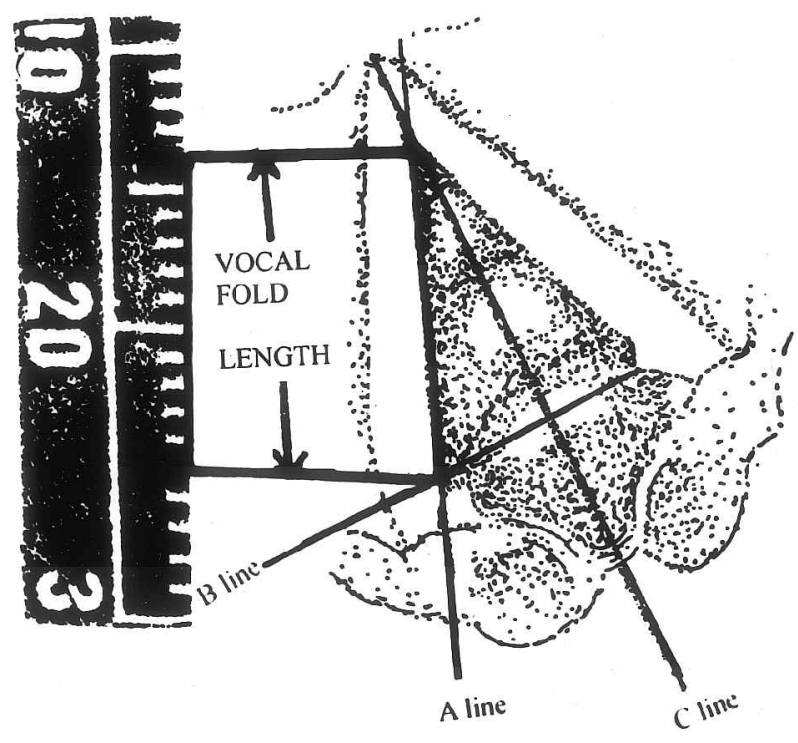

FIG. 1

Measurement of vocal fold length through direct laryngoscope. Right side: Sketch of the vocal fold. Line A: the inner border of the vocal process. Line B: the line connecting the vocal process of the two sides. Line $C$ : the line bisecting line $B$ perpendicularly. Left side: The vocal fold length measured with a comparable 'new scale'.

neuroleptic anaesthesia and in most cases under general anaesthesia, but if the process is obscure when examined, direct palpation was performed to confirm the position of process. Although in our study, the size/length of the arytenoid process was not measured, the variation in size/length of the process is not great from our experience. When the anterior commissure was not shown in the photograph, the cross point of lines $\mathrm{C}$ and $\mathrm{A}$ was taken as a reference point. In the open phase, the vocal fold length was measured accordingly.

\section{Results}

The results are shown in Table I. The average length of the vocal fold of Taiwanese is $14.6 \pm 1.2 \mathrm{~mm}$ for the male, $11.1 \pm 1.0 \mathrm{~mm}$ for the female in the group undergoing neuroleptic anaesthesia, and $15.3 \pm 1.6 \mathrm{~mm}$ for the male, $13.5 \pm 1.3 \mathrm{~mm}$ for the female in the group undergoing general anaesthesia. The statistical analysis was performed with Student's $t$-test. It revealed significant differences between the sexes and the type of anaesthesia $(p<0.001$ between the sexes in both groups; and $p<0.001$ for female, $p<0.05$ for male for the two different types of anaesthesia).

\section{Discussion}

From literature searches, previously the length of the human vocal fold has been either measured from cadavers or estimated from X-ray films. ${ }^{1,2}$ The results obtained from cadavers could not reflect the physiological vocal fold length in a living subject, and therefore do not provide useful clinical information. Using radiotherapy for estimation of vocal fold length, a lateral X-ray film of the neck is taken. The vague landmark in the film is used to estimate the vocal fold length. Although this method is convenient and gives practical data, it is difficult to measure the vocal fold length precisely. In lateral radiography, the thickness of the vocal fold renders the reference points obscure and makes interpretation of the data difficult. The present study gives a more precise measurement of the vocal fold length in a living subject. A photograph of the vocal fold was taken under direct laryngoscopy and then the vocal fold was measured with reference to a comparable new scale.

Physiologically, the vocal fold length changes in accordance with numerous situations, e.g. the pitch of the voice, the intensity of phonation, the shape and the size of the glottic closure area. ${ }^{3,4}$ In our study, we have chosen the open phase in which the length of the out-stretched vocal fold could be seen clearly through the direct laryngoscope. The measurement of the vocal fold length was performed in a routine procedure thereby avoiding discrepancy in data interpretation. We studied elongation of the vocal fold under general anaesthesia versus neuroleptic anaesthesia. It may be speculated that the difference originates from the loss of muscle tone or stretching by the endotracheal tube insertion or perhaps due to the laryngoscope in the endolarynx. Either way, that would change the 3-D shape of the vocal fold. This would cause thinning of the vocal fold and widening of the base of the benign lesion. This factor should be taken into consideration when performing functional surgery of the vocal fold under general anaesthesia.

In conclusion, we have developed a simple method for measuring the length of the human vocal fold in vivo. The results revealed differences in vocal fold length between general anaesthesia and neuroleptic anaesthesia. Elongation of the vocal fold under general rather than neuroleptic anaesthesia may be important when performing functional surgery of the vocal fold.

TABLE I

THE LENGTH OF VOCAL FOLD IN TAIWANESE ADULTS (MM)

\begin{tabular}{lccc}
\hline & General anaesthesia & Neuroleptic anaesthesia & $p$ value \\
\hline Males & $15.3 \pm 1.6(\mathrm{n}=23)$ & $14.6 \pm 1.2$ & $<0.05$ \\
Females & $13.5 \pm 1.3(\mathrm{n}=42)$ & $11.1 \pm 1.0$ & $<0.001$ \\
$p$ value & $<0.001$ & $<0.001$ & \\
\hline
\end{tabular}




\section{Acknowledgement}

This research was supported by the National Science Council Republic of China (NSC-89-2314-B-002225).

\section{References}

1 Run WN, Chung YS. Roentgenological measurement of physiological vocal cord length. Folia Phoniatr 1983;35:289-93

2 Damste PH, Hollien H, Moore P, Murry T. An X-ray study of vocal fold length. Folia Phoniatr 1968;20:349-59

3 Hollien H, Brown WS Jr, Hollien K. Vocal fold length associated with modal, falsetto and varying intensity phonations. Folia Phoniatr 1971;23:66-78

4 Hollien H, Damste H, Murry T. Vocal fold length during vocal fry phonation. Folia Phoniatr 1969;21:257-65
Address for correspondence:

Shiann-Yann Lee, M.D.,

Department of Otolaryngology,

National Taiwan University Hospital,

7 Chung-Shan South Road,

Taipei,

Taiwan 100.

Fax: +886-2-23410905

E-mail: yth@ha.mc.ntu.edu.tw

Dr Mao-Chang Su takes responsibility for the integrity of the content of the paper.

Competing interests: None declared 